\title{
Service Robots Adaptive Mutual-coupled Immune Network Planning Algorithm Research Based on the Distance-weighted
}

\author{
Rong Chen ${ }^{1 *}$, Hutian Feng ${ }^{2}$ \\ School of Mechanical Engineering, Nanjing University of Science \& Technology, Nanjing 210094, China. \\ * Corresponding author. Tel: +8618251968273; email: chenrong_njust@163.com \\ Manuscript submitted May 14, 2016; accepted January 5, 2017. \\ doi: $10.17706 /$ jcp.13.1.58-68
}

\begin{abstract}
In order to further improve efficiency and accuracy of the multi-service robot path planning in a complex and uncertain environment, based on the mutual-coupled immune network planning algorithm, an improved measurement algorithm was proposed. According to the antigen information of obstacles and the target, situation-oriented and goal-oriented coupling immune network are defined respectively and the weight coefficient is used to control their roles in the overall behavior. In order to further improve the planning performance of the robot, weight coefficient is defined dynamically according to the distance of the robot apart from the obstacles and the target. Thus we are able to optimize the robot's behavior by choosing the obstacle-avoidance behavior or the tend-to-target behavior in real time. The test results in the static multi-obstacle environment indicate that the algorithm proposed in this paper is more effective than other algorithms in literature, both in the aspects of length and smoothness, which verifies the ability of the algorithm to improve the efficiency and precision of the path planning. And the multi-robot can successfully avoid dynamic obstacles and achieve the targeting result according to the test results in the complex and uncertainty environment, which verifies its flexibility and robustness.
\end{abstract}

Key words: Service robot, path planning, distance weighting, adaptive, immune network.

\section{Introduction}

Path planning is one of the prerequisites with which service robots complete all kinds of services [1]. The path planning problem of multi-service robot has always been a hot research topic in the field of robot [2]-[5]. Many researchers are perspective to the multi-robot coordinated control, because multi-robot system has more advantages than single robot system. The multi-robot path planning, due to its complexity, causes the attention of many researchers. For example, Multi-robot coordinated path planning in unknown dynamic environment was proposed by Zheng Taixiong and Wang Ping [6]. The activity space of robot is decomposed into several sub-planning zones dynamically, and then the algorithm dynamically chooses the next path planning in each sub-planning zone according to the detected environmental information. Thus, the path planning in unknown environment is achieved. Cooperative simulated annealing for path planning in multi-robot systems was proposed by Gildardo Sanchez-Ante. et al. [7]. Its main idea is that each robot generates path respectively and then all the robot's paths are coordinated, which achieves the multi-robot path planning.

The immune system can maintain the stability of internal environment with lymphocytes. In recent years, 
some characteristics of the immune system have attracted the attention of researchers, and some algorithms based on the immune system have been widely applied in the robot path planning. Multi-objective path planning for space exploration robot based on chaos immune particle swarm optimization algorithm was proposed by Hao Wei and Qin Shi [8], which improves the search speed and can avoid obstacles and plan path better. Artificial immune system based path planning of mobile robot was proposed by P.K. Das. et al [9]. The mobile robot based on the algorithm can effectively avoid obstacles and get out from the "dead zone", and then completes the path planning task. Mobile robot path planning based on improved ant colony algorithm was proposed by Pan Jie et al [10]. It can be seen that unnecessary spikes in path are eliminated by using this algorithm to plan path. Moreover, the smoothness of the path is better and The length of the path is shorter. Mobile robot path finding and planning algorithm based on the artificial immune network was proposed by Zhuang Jian and Wang Sunan [11]. Experimental results of the algorithm show that the algorithm has a good quickness and flexibility, which enables robots finish different tasks in a short time. In addition, a mathematical proof for the algorithm's convergence is given based on the theory of Markov Chain. Ishiguro A et al [12] put forward an immunological approach to dynamic behavior control for autonomous mobile robots. Based on the immune network, the concept of interconnected coupling was proposed, namely the planning network is divided into two kinds called situation-oriented immune network and goal-oriented immune network. As a result, robots choose the appropriate antibodies to avoid obstacles and approach the goal under the excitation of antigen information of obstacles and the target, as well as the stimulation and inhibition between antibodies, which solves the problem of path choice better. However, the definition of antibody is relatively simple, it only considers whether there are obstacles on the detection direction, so the algorithm doesn't reach the optimal effect of planning. Therefore, according to the detection distance, the antibodies are redefined based on the mutual-coupled immune network planning model, and the dynamic weight coefficient is set up to control the impact that obstacle-avoidance behavior and tend-to-target behavior had on the robot overall behavior. The validity of the algorithm was verified by the test results in a static and dynamic environment.

\section{Simple Mutual-coupled Immune Network Planning Model}

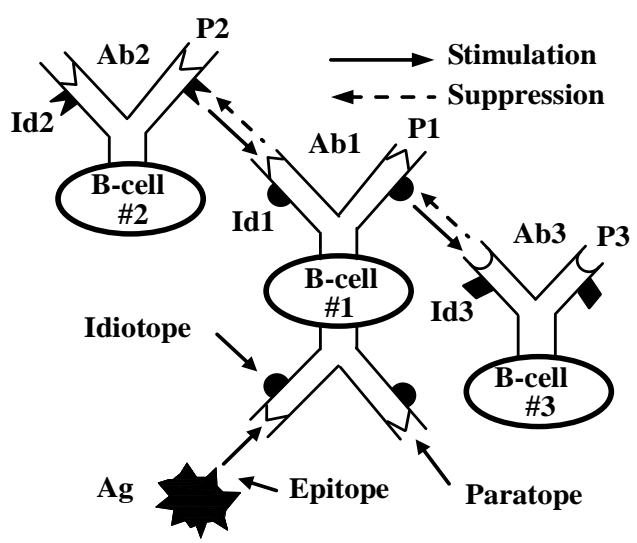

Fig. 1. The hypothesis of idiotypic immune network.

Immunization is a kind of specific physiological response of the body. Immune system recognizes and eliminates the antigenicity foreign body by the lymphocytes which distribute in the whole body, and then maintains the stability of internal environment. The idiotypic immune network theory was proposed by Jerne [13]. As shown in Fig. 1, when the antigen invades, it will be recognised by the paratope of B - cell \# 1 which stimulates the cells to produce large amounts of antibodies to clear antigens. Similarly, B - cell \# 1 Proliferates, differentiates and then produces antibodies that are equal to the antigens for B - cell \# 2 and B - 
cell \# 3. The Id1 stimulates B - cell \# 2 by stimulating paratope P2, so B - cell \# 2 can also produce antibodies to inhibit B - cell \# 1 antibody production. B - cell through such stimulation, inhibition forms a large-scale network to identify and eliminate the dissent.

In order to make service robot rapidly perceive the external environment and do the right judgment, a behavior arbitration mechanism is needed for the robot to determine the movement. In this paper, an artificial immune agent is built based on the hypothesis of idiotypic immune network. The current working environment is made as antigens, and the individual behavior element is made as antibodies. There are eight virtual sensors around the service robot, that is, there are eight detection directions D. Robot can move along the eight directions $\mathrm{M}, \mathrm{D}$ and $\mathrm{M}$ are in the same direction. Namely $\mathrm{M}=\mathrm{D}=\left\{\mathrm{L} 90^{\circ}, \mathrm{L} 60^{\circ}, \mathrm{L} 30^{\circ}\right.$, Front, $\mathrm{R} 30^{\circ}$, $\left.\mathrm{R} 60^{\circ}, \mathrm{R} 90^{\circ}, \mathrm{Back}\right\}$. According to the environment detected by sensors, service robot prompts $\mathrm{B}$ cells to produce corresponding antibodies (the behavior of service robot). Service robot can move along the eight directions. Namely \{turn left 90 , turn left 60 , turn left 30 , move forward, turn right 30 , turn right 60 , turn right 90 , move back\}. When service robot reaches the target point, it will stop moving, and the task is completed at this time.

Because the environment which the robot is in is complex and uncertain, a separate network cannot meet the requirement of the planning. Here, mutual-coupled immune network planning model is presented, namely the planning network is divided into two kinds called situation-oriented immune network and goal-oriented immune network. As shown in Fig. 2.



Fig. 2. Mutual-coupled immune network of service robot.

\section{Service Robots Adaptive Mutual-coupled Immune Network Planning Algorithm Based on the Distance-weighted}

\subsection{Re-definition of Antibodies}

Antibody means the behavior that service robots choose according to the environment detected by sensors. Antibody can be defined as a combination of epitope and paratope. The premise of behavior and the taken action are made as a paratope, while the epitope part expresses the interaction relationship and strength Mij between antibodies. According to mutual-coupled immune network planning model, antibodies include two kinds that are situation-oriented and goal-oriented. In order to further improve the performance of network planning, in this paper, the distance detected by robot's sensors can be divided into far, middle, near, and $\{01,10,11\}$ is used to encode with binary. If an obstacle is over the distance that robot's sensors can detect, use 00 to encode. According to the environmental information and the robot's behavior, 22 groups of antibodies are defined in this paper, which are shown in Table 1 and Table 2. In the table, \# represents that the information is not important, and 0 or 1 could be used to replace it.

Take antibody 6 for example, it can be seen from the Table $1 \& 2$ that the antibody turns right $30^{\circ}$. In the situation-oriented network, the requirements of the antibody are as follows: In robot $\mathrm{L} 30^{\circ}$ direction, having 
the middle-distance obstacles; In the front, having the close obstacles; In R30 ${ }^{\circ}$ direction, having the long-distance obstacles; In $\mathrm{L} 90^{\circ}, \mathrm{L} 60^{\circ}, \mathrm{R} 60^{\circ}, \mathrm{R} 90^{\circ}$ and rear directions, obstacles are dispensable; The target is in any direction. In the goal-oriented network, the requirements of the antibody are as follows: In robot R30 direction, having long-distance obstacles; In other directions, the obstacles are dispensable; The target is in the front.

Table 1. The Definition of Situation-Oriented Antibody

\begin{tabular}{llllllllllll}
\hline \hline Antiobody No. & \multicolumn{1}{c}{ Precondition of obstacle avoidance (Paratope) } & & Behavior \\
\hline Antiobody 1 & $\# \#$ & $\# \#$ & $\# \#$ & 00 & $\# \#$ & $\# \#$ & $\# \#$ & $\# \#$ & $\#$ & $\#$ & move forward \\
Antiobody 2 & $\# \#$ & $\# \#$ & $\# \#$ & 01 & 01 & $\# \#$ & $\# \#$ & $\# \#$ & $\#$ & $\#$ & move forward \\
Antiobody 3 & $\# \#$ & 01 & 00 & 10 & 00 & 01 & $\# \#$ & $\# \#$ & $\#$ & $\#$ & move forward \\
Antiobody 4 & 11 & 11 & 11 & 11 & 11 & 11 & 11 & 00 & $\#$ & $\#$ & move back \\
Antiobody 5 & $\# \#$ & $\# \#$ & $\# \#$ & 11 & 00 & $\# \#$ & $\# \#$ & $\# \#$ & $\#$ & $\#$ & turn right 30 \\
Antiobody 6 & $\# \#$ & $\# \#$ & 10 & 11 & 01 & $\# \#$ & $\# \#$ & $\# \#$ & $\#$ & $\#$ & turn right 30 \\
Antiobody 7 & $\# \#$ & 00 & 01 & 01 & 10 & $\# \#$ & $\# \#$ & $\# \#$ & $\#$ & $\#$ & turn right 30 \\
Antiobody 8 & $\# \#$ & $\# \#$ & 11 & 11 & 11 & 00 & $\# \#$ & $\# \#$ & $\#$ & $\#$ & turn right 60 \\
Antiobody 9 & $\# \#$ & $\# \#$ & 10 & 11 & 10 & 01 & $\# \#$ & $\# \#$ & $\#$ & $\#$ & turn right 60 \\
Antiobody 10 & $\# \#$ & $\# \#$ & 11 & 10 & 01 & 10 & 99 & $\# \#$ & $\#$ & $\#$ & turn right 60 \\
Antiobody 11 & $\# \#$ & 11 & 11 & 11 & 11 & 11 & 00 & $\# \#$ & $\#$ & $\#$ & turn right 90 \\
Antiobody 12 & $\# \#$ & 01 & 10 & 11 & 01 & 10 & 01 & $\# \#$ & $\#$ & $\#$ & turn right 90 \\
Antiobody 13 & $\# \#$ & 00 & $\# \#$ & 10 & 01 & 10 & 10 & 00 & $\#$ & $\#$ & turn right 90 \\
Antiobody 14 & $\# \#$ & $\# \#$ & 00 & 11 & $\# \#$ & $\# \#$ & $\# \#$ & $\# \#$ & $\#$ & $\#$ & turn left 30 \\
Antiobody 15 & $\# \#$ & $\# \#$ & 01 & 10 & 11 & $\# \#$ & $\# \#$ & $\# \#$ & $\#$ & $\#$ & turn left 30 \\
Antiobody 16 & $\# \#$ & 01 & 10 & 01 & $\# \#$ & $\# \#$ & 10 & $\# \#$ & $\#$ & $\#$ & turn left 30 \\
Antiobody 17 & $\# \#$ & 00 & 11 & 11 & 11 & $\# \#$ & $\# \#$ & $\# \#$ & $\#$ & $\#$ & turn left 60 \\
Antiobody 18 & $\# \#$ & 01 & 01 & 11 & 10 & $\# \#$ & $\# \#$ & $\# \#$ & $\#$ & $\#$ & turn left 60 \\
Antiobody 19 & $\# \#$ & 10 & 00 & 10 & 01 & $\# \#$ & 00 & $\# \#$ & $\#$ & $\#$ & turn left 60 \\
Antiobody 20 & 00 & 11 & 11 & 11 & 11 & 11 & $\# \#$ & $\# \#$ & $\#$ & $\#$ & turn left 90 \\
Antiobody 21 & 01 & 01 & 10 & 11 & 11 & 10 & $\# \#$ & $\# \#$ & $\#$ & $\#$ & turn left 90 \\
Antiobody 22 & 10 & $\# \#$ & $\# \#$ & 10 & 11 & 01 & $\# \#$ & 00 & $\#$ & $\#$ & turn left 90 \\
\hline \hline
\end{tabular}

Table 2. The Definition of Goal-Oriented Antibody

\begin{tabular}{|c|c|c|c|c|c|c|c|c|c|c|c|}
\hline Antiobody No. & \multicolumn{10}{|c|}{ Precondition of goal approach (Paratope) } & \multirow{2}{*}{$\begin{array}{l}\text { Behavior } \\
\text { move forward }\end{array}$} \\
\hline Antiobody 1 & \#\# & \#\# & \#\# & 00 & \#\# & \#\# & \#\# & \#\# & 4 & \# & \\
\hline Antiobody 2 & \#\# & \#\# & \#\# & 01 & \#\# & \#\# & \#\# & \#\# & 4 & \# & move forward \\
\hline Antiobody 3 & \#\# & \#\# & \#\# & 10 & \#\# & \#\# & \#\# & \#\# & 4 & \# & move forward \\
\hline Antiobody 4 & \#\# & \#\# & \#\# & \#\# & \#\# & \#\# & \#\# & 00 & 8 & \# & move back \\
\hline Antiobody 5 & \#\# & \#\# & \#\# & \#\# & 00 & \#\# & \#\# & \#\# & 5 & \# & turn right 30 \\
\hline Antiobody 6 & \#\# & \#\# & \#\# & \#\# & 01 & \#\# & \#\# & \#\# & 5 & \# & turn right 30 \\
\hline Antiobody 7 & \#\# & \#\# & 10 & \#\# & 10 & \#\# & \#\# & 00 & 5 & \# & turn right 30 \\
\hline Antiobody 8 & \#\# & \#\# & \#\# & \#\# & \#\# & 00 & \#\# & \#\# & 6 & \# & turn right 60 \\
\hline Antiobody 9 & \#\# & \#\# & \#\# & 10 & \#\# & 01 & \#\# & \#\# & 6 & \# & turn right 60 \\
\hline Antiobody 10 & \#\# & \#\# & 11 & \#\# & \#\# & 10 & \#\# & 01 & 6 & \# & turn right 60 \\
\hline Antiobody 11 & \#\# & \#\# & \#\# & \#\# & \#\# & \#\# & 00 & \#\# & 7 & \# & turn right 90 \\
\hline Antiobody 12 & \#\# & \#\# & \#\# & \#\# & \#\# & 10 & 01 & \#\# & 7 & \# & turn right 90 \\
\hline Antiobody 13 & \#\# & \#\# & 10 & 11 & \#\# & \#\# & 10 & \#\# & 7 & \# & turn right 90 \\
\hline Antiobody 14 & \#\# & \#\# & 00 & \#\# & \#\# & \#\# & \#\# & \#\# & 3 & \# & turn left 30 \\
\hline Antiobody 15 & \#\# & 01 & 01 & 11 & \#\# & \#\# & \#\# & \#\# & 3 & \# & turn left 30 \\
\hline Antiobody 16 & \#\# & \#\# & 10 & \#\# & \#\# & 01 & \#\# & \#\# & 3 & \# & turn left 30 \\
\hline Antiobody 17 & \#\# & 00 & \#\# & 10 & \#\# & \#\# & \#\# & \#\# & 2 & \# & turn left 60 \\
\hline Antiobody 18 & \#\# & 01 & \#\# & \#\# & \#\# & \#\# & 01 & \#\# & 2 & \# & turn left 60 \\
\hline Antiobody 19 & \#\# & 10 & \#\# & \#\# & \#\# & 10 & 00 & \#\# & 2 & \# & turn left 60 \\
\hline Antiobody 20 & 00 & \#\# & \#\# & \#\# & \#\# & \#\# & \#\# & \#\# & 1 & \# & turn left 90 \\
\hline Antiobody 21 & 01 & \#\# & 10 & \#\# & \#\# & 10 & \#\# & \#\# & 1 & \# & turn left 90 \\
\hline Antiobody 22 & 10 & \#\# & \#\# & 11 & 10 & \#\# & \#\# & 00 & 1 & $\#$ & turn left 90 \\
\hline
\end{tabular}

\subsection{Selection of Antibodies}

The calculation of antibody concentration is based on the dynamic model of Farmer. In this paper, antibodies are divided into two kinds that are situation-oriented and goal-oriented, so when calculate the concentration of antibodies, we should calculate the concentration of situation-oriented antibodies and 
goal-oriented antibodies respectively, and then sum. Take the calculation of the situation-oriented antibody concentration for example, the calculation of the goal-oriented antibody concentration is similar.

$$
\begin{aligned}
& \frac{d a_{i}^{o}(t)}{d t}=\left(\alpha_{1} \frac{\sum_{j \neq i, j=1}^{N} m_{j i}^{o} a_{j}^{o}(t-1)}{N}-\alpha_{2} \frac{\sum_{k \neq i, k=1}^{N} m_{i k}^{o} a_{k}^{o}(t-1)}{N}+\beta m_{i}^{o}-k_{i}^{o}\right) \times a_{i}^{o}(t-1) \\
& S_{i}^{o}(t)=a_{i}^{o}(t-1)+\frac{d a_{i}^{o}(t)}{d t} \\
& a_{i}^{o}(t)=\frac{1}{1+\exp \left(0.5-S_{i}^{o}(t)\right)}
\end{aligned}
$$

In the type (1), $N$ shows the number of antibodies, $\alpha_{1}, \alpha_{2}, \beta$ are adjustment coefficients, $m_{j i}{ }^{o}$ shows the affinity between antibody $j$ and antibody $i$, and $m_{i}{ }^{o}$ shows the affinity between antibody $i$ and antigen. The first and the second in the right parenthese of type (1) show stimulation and inhibition that other antibodies have on antibody $i$. The third represents the stimulation of antigen. The fourth $k_{i}{ }^{o}$ represents the natural death of antibodies. Type (3) is used to ensure the stability of antibody concentration and normalize the antibody concentration. The specific calculation formulas are as follows:

$$
\begin{aligned}
m_{j i}^{o} & =\sum_{t=1}^{N} \sum_{l=2 t-1}^{2 t} p_{j}^{o}(l) \oplus \overline{p_{i}^{o}(l)} \\
m_{i}^{o} & =\sum_{t=1}^{N} \sum_{l=2 t-1}^{2 t} e^{o}(l) \oplus \overline{p_{i}^{o}(l)} \\
m_{i}^{g} & =\sum_{t=1}^{N} \sum_{l=2 t-1}^{2 t} e^{g}(l) \wedge \overline{p_{i}^{g}(l)}
\end{aligned}
$$

where, $\oplus$ shows XOR operation; $\wedge$ shows OR operation; $p_{j}^{o}(I)$ and $p_{i}{ }^{o}(I)$ respectively show inhibitory effect that the pheromone on paratope of antibody $j$ has on antibody $i ; e^{o}(I)$ shows stimulation effect that pheromone of antigen has on antibody $i$.

Robot path planning is a result that the obstacle-avoidance behavior and the tend-to-target behavior work together [14]. The impact which some behavior has on overall behavior is defined by the weight coefficient. The environment is complex and uncertain, if the weight coefficient is fixed, it cannot meet the needs of the navigation very well. Therefore, with the change of the robot's position, weight coefficient changes dynamically to optimize the robot's behavior by choosing the obstacle-avoidance behavior or the tend-to-target behavior in real time, and then make robots plan out a better path. This function embodies the competition relation of the two behavior patterns, it can be described by the following mathematical model:

$$
\begin{aligned}
& w_{g}=\alpha_{1} w_{1}\left(1-w_{1}^{2}\right)-\gamma_{12} w_{1} w_{2}^{2} \\
& w_{o}=\alpha_{2} w_{2}\left(1-w_{2}^{2}\right)-\gamma_{21} w_{2} w_{1}^{2}
\end{aligned}
$$

where, $w_{1}, w_{2}$ respectively show the initial weight coefficient of goal-oriented and situation-oriented immune network. $\alpha_{1}, \alpha_{2}, \gamma_{12}, \gamma_{21}$ are the parameters related to the environment and change with the change of mobile robot's location. $\alpha_{1}$ shows a competitive advantage of tend-to-target behavior, $\alpha_{2}$ shows a competitive advantage of obstacle-avoidance behavior, $\gamma_{12}$ shows a restriction degree that obstacle-avoidance behavior has on tend-to-target behavior, $\gamma_{21}$ shows a restriction degree that tend-to-target behavior has on obstacle-avoidance behavior. The specific calculation steps are as follows:

$$
\alpha_{2}=\sum e^{-d_{i} / d_{1}} / n
$$




$$
\begin{aligned}
& \alpha_{1}=k_{1}\left(1-\alpha_{2}\right) \\
& \gamma_{21}=k_{2} e^{-d_{r g} / d_{2}} \\
& \gamma_{12}=k_{3}\left(1-\gamma_{21}\right)
\end{aligned}
$$

where, $d_{1}, d_{2}$ are correlation coefficients; $k_{1}, k_{2}, k_{3}$ are adjustment coefficients; $d_{i}$ shows the distance between the robot and obstacles; $d_{r g}$ shows the distance between the robot and the target point.

Calculation formula of the total concentration of antibody is:

$$
a_{i}=w_{o} * a_{i}^{o}+w_{g} * a_{i}^{g}
$$

\subsection{Steps of the Algorithm}

\section{Begin:}

Initialize: adjustment coefficients $\alpha_{1}, \alpha_{2}, \beta, k_{1}, k_{2}, k_{3}$; correlation coefficients $d_{1}, d_{2}$; initial weight coefficients $w_{1}$, $w_{2}$; natural mortality $k_{i}$.

Set up the environment with obstacles.

While: antigen invades, do

Select the antibody set: $A(k)$;

Calculate antibodies stimulus value: $a^{g}=\frac{d A^{g}(k)}{d t}, a^{o}=\frac{d A^{o}(k)}{d t}$;

Calculate the antibody concentration:

$$
A^{g}(k)=\frac{1}{1+\exp \left(0.5-S^{g}(k)\right)}, \quad A^{o}(k)=\frac{1}{1+\exp \left(0.5-S^{o}(k)\right)}, a(k)=w_{g} * A^{g}(k)+w_{o} * A^{o}(k) ;
$$

Choose antibodies: $\max (a(k))$;

Determine the robot's behavior.

End

End

\section{Simulation Results Analysis and Discussion}

In order to verify the effectiveness of the proposed algorithm (DW-AMINPA), path planning for a single robot in four different static environments and two robots in two different dynamic environments are tested by using Matlab 7.0.1 on PIV 2.53 GHz 2 GB memory computer, and the results are compared with the testing results of simple mutual-coupled immune network planning algorithm (SMINPA) and the algorithm which antibodies are redefined based on the mutual-coupled immune network planning algorithm (AR-MINPA).

\subsection{Test in the Static Environment}

The parameters of SMINPA are set to: $\alpha=0.01, \beta=0.5, \gamma=0.5, k_{i}=0.05$; The parameters of AR-MINPA are set to: $\alpha=0.01, \beta=0.5, \gamma=0.5, k_{i}=0.05$; The parameters of DW-AMINPA are set to: $\alpha=0.01, \beta=0.1, k_{i}=0.05, w_{1}=w_{2}=0.5$, $k_{1}=0.5, k_{2}=10, k_{3}=0.01, d_{1}=0.2, d_{2}=0.5$.

Fig. 3 to Fig. 6 show the simulation results of the three algorithms in four different static environments. From the figure, it can be seen that robots based on the three algorithms can find their own path from the starting point START to the end GOAL in four different static environments. But from the point of the path of planning, compared with the robots based on other two kinds of algorithms, planned path of the robots based on the proposed algorithm is shorter, the turning angle is smaller, and shows better smoothness. Thus, planning efficiency and precision of the DW-AMINPA are successfully verified. 


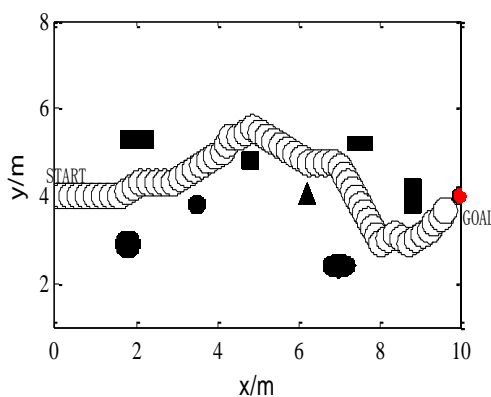

(a) SMINPA

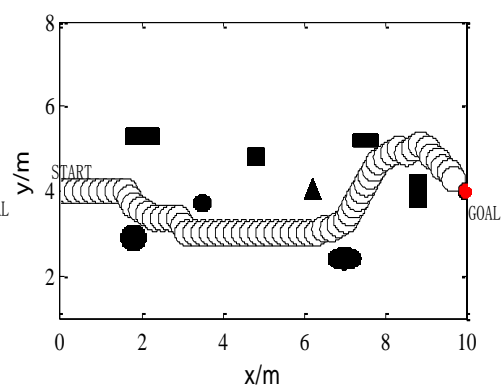

(b) AR-MINPA

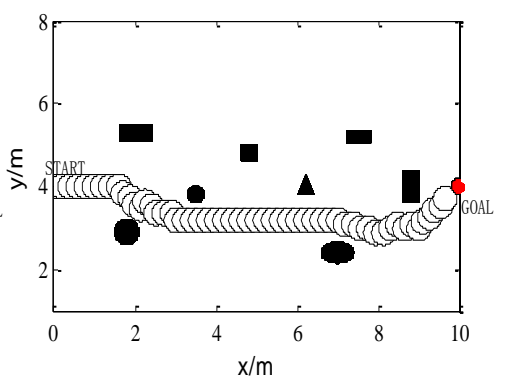

(c) DW-AMINPA

Fig. 3. Planning comparisons in the static environment 1.



(a) SMINPA

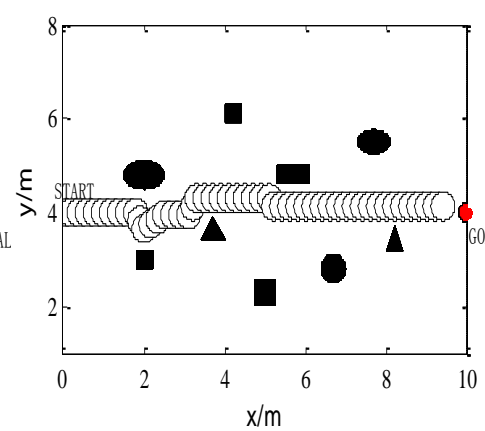

(b) AR-MINPA

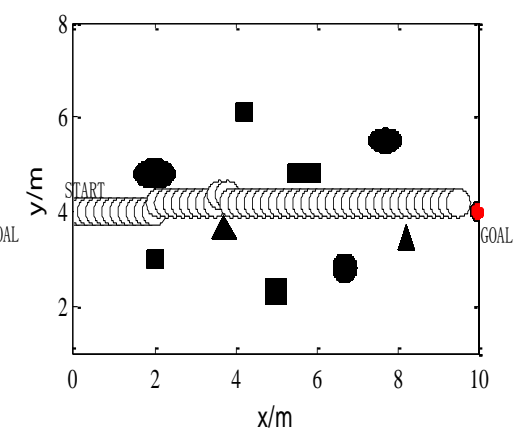

(c) DW-AMINPA

Fig. 4. Planning comparisons in the static environment 2.

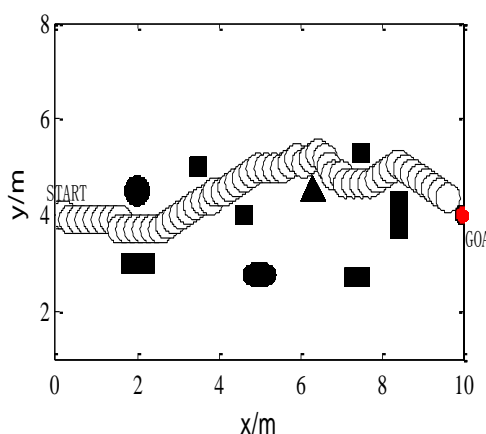

(a) SMINPA

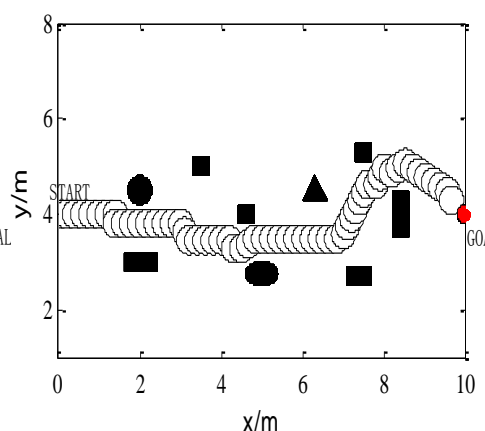

(b) AR-MINPA

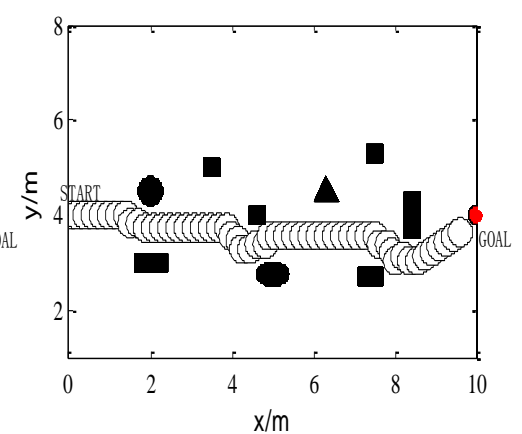

(c) DW-AMINPA

Fig. 5. Planning comparisons in the static environment 3.



(a) SMINPA

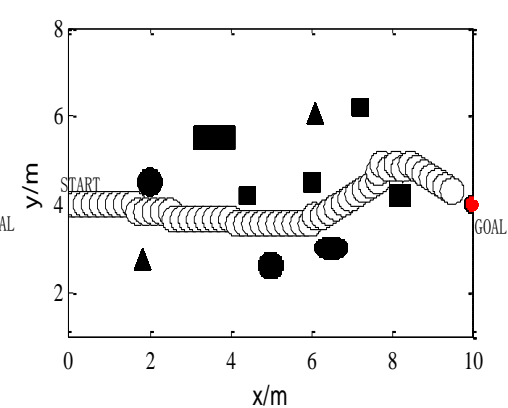

(b) AR-MINPA

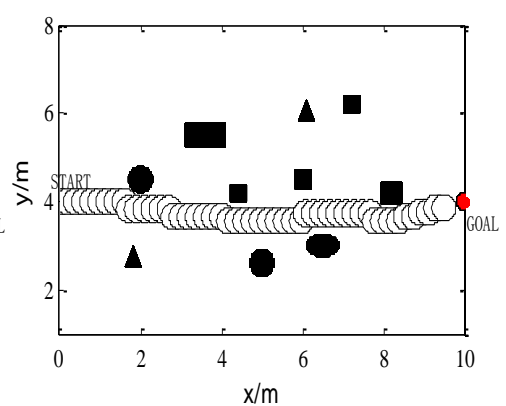

(c) DW-AMINPA

Fig. 6. Planning comparisons in the static environment 4.

Table 3. Planning Performance Comparisons of Three Algorithms in Four Kinds of Environments

\begin{tabular}{lllllll}
\hline \hline \multirow{2}{*}{ Environment } & \multicolumn{3}{l}{ Length of planned path } & \multicolumn{3}{l}{ Smoothness of planned path } \\
\cline { 2 - 7 } & SMINPA & AR-MINPA & DW-AMINPA & SMINPA & AR-MINPA & DW-AMINPA \\
\hline 1 & 11.8 & 11.6 & 10.8 & $11.19^{\circ}$ & $10.34^{\circ}$ & $9.11^{\circ}$ \\
2 & 10.4 & 10 & 9.8 & $9.2^{\circ}$ & $7.2^{\circ}$ & $3.67^{\circ}$ \\
3 & 11 & 11.4 & 10.6 & $19.09^{\circ}$ & $12.63^{\circ}$ & $11.89^{\circ}$ \\
4 & 10.6 & 10.6 & 10 & $14.15^{\circ}$ & $12.45^{\circ}$ & $7.2^{\circ}$ \\
\hline \hline
\end{tabular}


In order to see the superiority of the proposed algorithm more clearly, the length and smoothness of planned path of the three algorithms are also tested, and the results are shown in table 3. From table 3, it can be seen that planning performance of SMINPA is poorer, while planning performance of AR-MINPA is better than that of SMINPA in four different static environments. This is because AR-MINPA according to the detection distance redefines the antibodies based on the SMINPA, which makes antibodies more intelligent, and then robots can make a behavioral decision suitable for environments. Compared with SMINPA and AR-MINPA, the length of planned path of DW-AMINPA is shorter, and smoothness is better. This is mainly due to the changing weight coefficient, which can judge the contribution that current obstacle-avoidance behavior and tend-to-target behavior make to overall behavior of antibodies, so that the robot chooses the appropriate behavior in real time. Therefore the accuracy and efficiency of the proposed algorithm are improved.

\subsection{Test in the Dynamic Environment}

In order to validate that the proposed algorithm can also plan out a feasible path in a dynamic uncertain environment, two different dynamic environments and two robots are set up in this paper. The parameters of the dynamic environment are shown in Table 4.

Table 4. Parameters of the Dynamic Environment

\begin{tabular}{|c|c|c|c|c|c|c|c|}
\hline \multirow[t]{2}{*}{ Parameters } & \multirow[t]{2}{*}{ Robots } & \multirow[t]{2}{*}{ Target points } & \multicolumn{3}{|c|}{$\begin{array}{l}\text { Dynamic obstacles in } \\
\text { environment1 }\end{array}$} & \multicolumn{2}{|c|}{$\begin{array}{l}\text { Dynamic obstacles in } \\
\text { environment } 2\end{array}$} \\
\hline & & & D_obs1 & D_obs2 & D_obs3 & D_obs1 & D_obs2 \\
\hline Location $\left[x_{\mathrm{p}}, y_{p}\right]^{T} / m$ & $\begin{array}{l}{[0,1]^{T}} \\
{[0,7]^{T}}\end{array}$ & $\begin{array}{l}{[8,6]^{T}} \\
{[8,2]^{T}}\end{array}$ & {$[2,4.2]^{T}$} & {$[3.5,4.5]^{T}$} & {$[6,0.5]^{T}$} & {$[2,4]^{T}$} & {$[6,0.8]^{T}$} \\
\hline speed $\left[x_{v}, y_{v}\right]^{T} / m \cdot s^{-1}$ & {$[0,0.2]^{T}$} & {$[0,0]^{T}$} & {$[0,0.1]^{T}$} & {$[0,-0.075]^{T}$} & {$[0,0.12]^{T}$} & {$[0.07,0.06]^{T}$} & {$[-0.05,0.12]^{T}$} \\
\hline
\end{tabular}

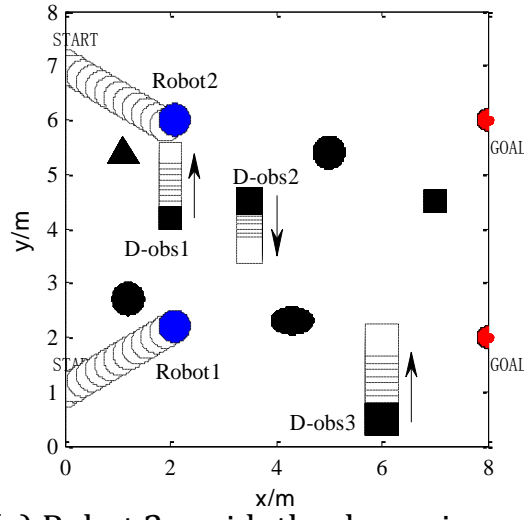

(a) Robot 2 avoids the dynamic obstacle 1

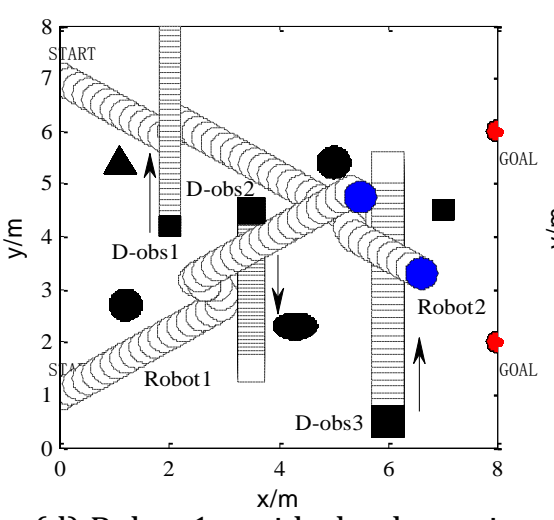

(d) Robot 1 avoids the dynamic obstacle 3

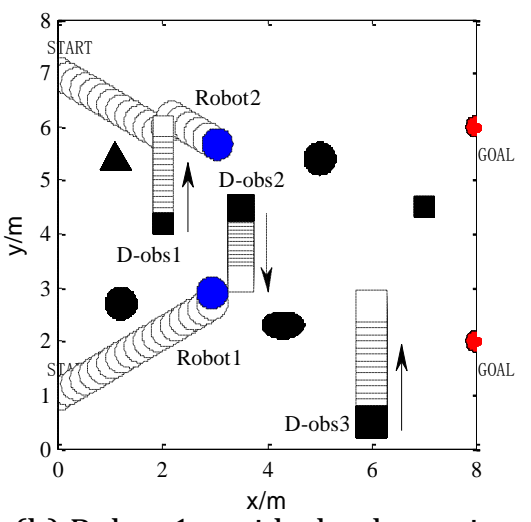

(b) Robot 1 avoids the dynamic obstacle 2

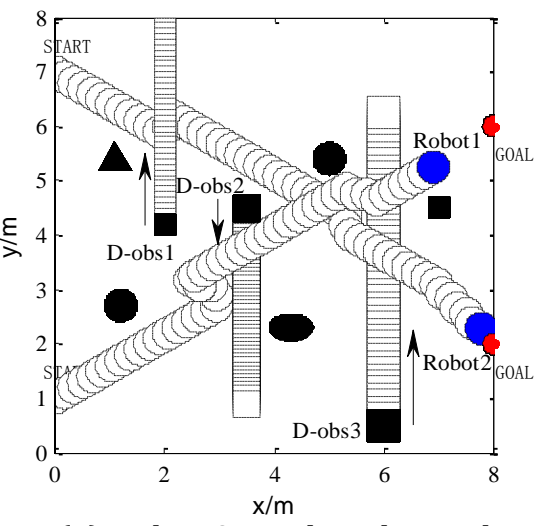

(e) Robot 2 reaches the goal successfully

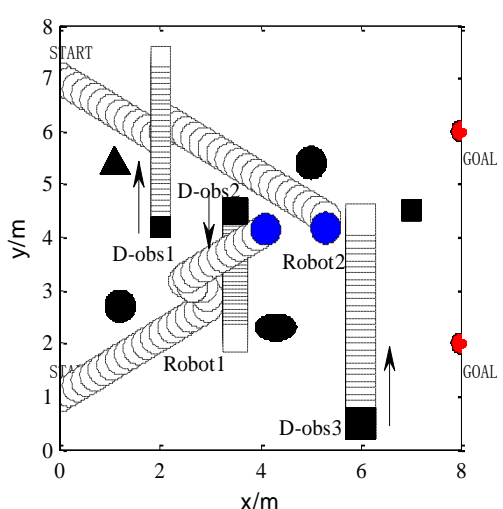

(c) Robot 2 avoids the dynamic obstacle 3

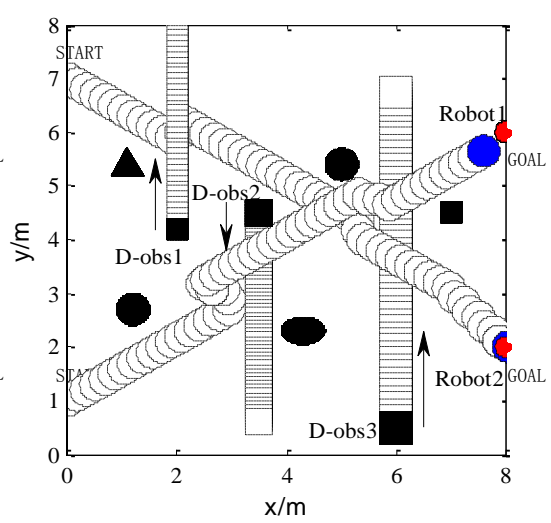

(f) Robot 1 reaches the goal successfully

Fig. 7. DW-AMINPA-based dynamic planning in the uncertain environment 1. 
As can be seen from the Fig. 7, robot $1 \& 2$ respectively start from the $[0,1] \mathrm{T}$ and $[0,7]$ T. Firstly, robot 2 meets the dynamic obstacle D_obs1. According to the current environmental information detected by robot 2 , the antibody concentration is calculated based on the proposed algorithm, and suitable antibodies are choosed through the controlling action of changing weight coefficient on two kinds of behaviors, and then robot makes the corresponding strategy so that robot 2 bypasses the D_obs1 successfully. These are shown in Fig. 7(a). Secondly, robot 1 meets the dynamic obstacle D_obs2. Robot 1 also bypasses and avoids the obstacle, and then moves to the target direction, which is shown in Fig. 7(b). Thirdly, robot 2 meets D_obs3, robot 1 meets D_obs3, and they all bypass the dynamic obstacles successfully, which is shown in Fig. 7(c) (d). Then robot 1, 2 meet the static obstacles, but they avoid the obstacles successfully and reach the goal through the suitable concentration calculation and selection of antibodies, as shown in Fig. 7(e) - (f).

As can be seen from the Fig. 8, robot $1 \& 2$ respectively start from the [0, 1]T and [0, 7]T . Firstly, robot 2 meets the dynamic obstacle D_obs1, then robot 1 encounters the dynamic obstacle D_obs2. They all succeed in bypassing obstacles, and then they continue to move on to the destination and arrive at the target site finally, which is shown in Fig. 8 (a) - (d).

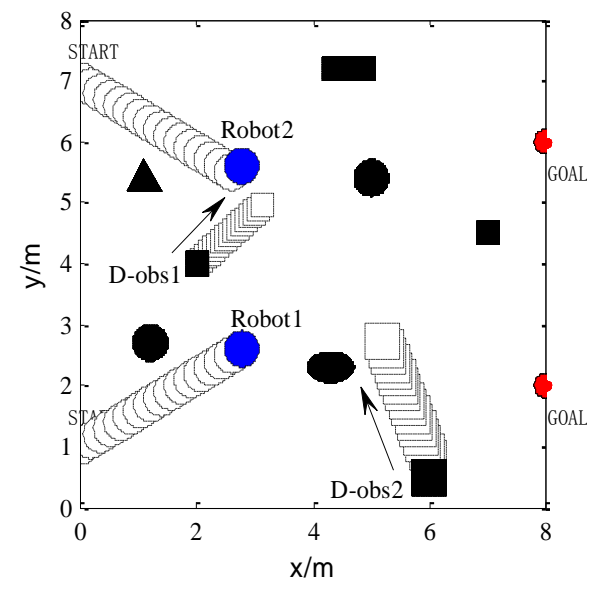

(a) Robot 2 avoids the dynamic obstacle 1

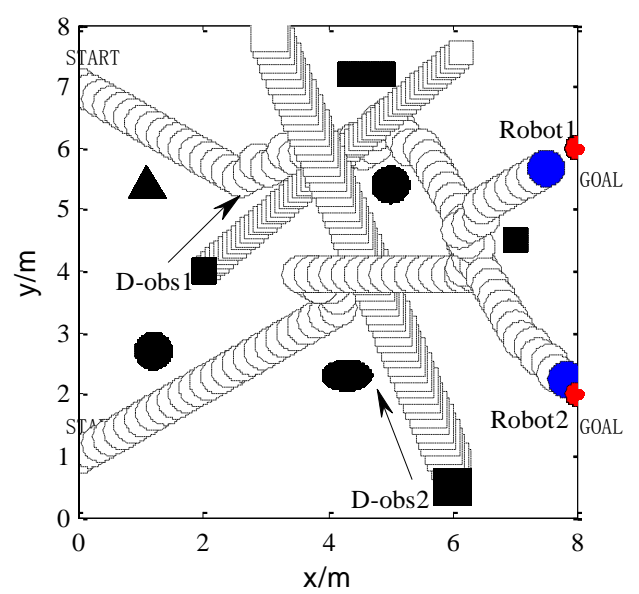

(c) Robot 2 reaches the goal successfully

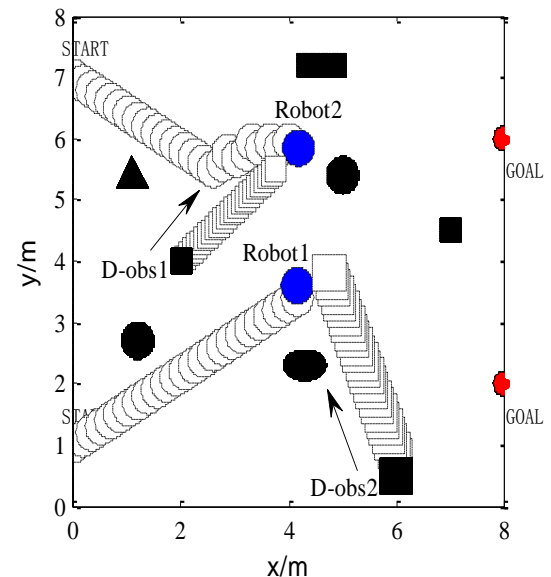

(b) Robot 1 avoids the dynamic obstacle 2

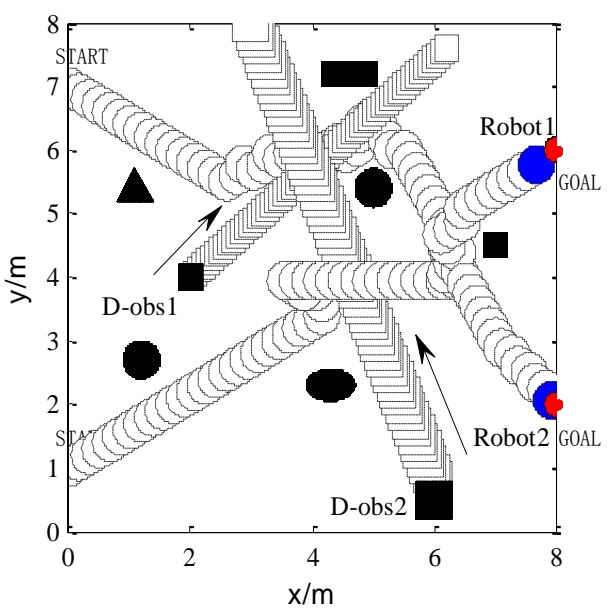

(d) Robot 1 reaches the goal successfully

Fig. 8. DW-AMINPA-based dynamic planning in the uncertain environment 2.

The time when each robot encounters the dynamic obstacles and arrives at the target are shown in Table 5 and Table 6:

Table 5. Time parameter in the dynamic environment 1

\begin{tabular}{lllllll}
\hline \hline Dynamic environment 1 & $a$ & $b$ & $c$ & $d$ & $e$ & $f$ \\
\hline
\end{tabular}




\begin{tabular}{lllllll}
\hline Time $(\mathrm{T} / \mathrm{s})$ & 12 & 18 & 32 & 40 & 48 & 52 \\
\hline \hline
\end{tabular}

Table 6. Time parameter in the dynamic environment 2

\begin{tabular}{lllll}
\hline \hline Dynamic environment 2 & $a$ & $b$ & $c$ & $d$ \\
\hline Time (T/s) & 16 & 25 & 58 & 60 \\
\hline \hline
\end{tabular}

Fig. 9 and Fig. 10 show planning results of SMINPA in the dynamic environment 1, 2. From Fig. 9, it can be seen that robot1 based on the SMINPA can escape D_obs2 successfully and robot2 based on the SMINPA can also escape D_obs1 successfully, but robot 2 couldn't avoid the dynamic obstacle D_obs3 very well. From Fig. 10 , it can be seen that robot1 based on the SMINPA escapes D_obs2 successfully, but when robot 2 meets D_obs1, it couldn't avoid very well. By comparing dynamic programming results of two planning algorithms, it can be seen that because the antibodies are redefined and changing weight coefficient is joined, the proposed algorithm has better flexibility and robustness, the overall planning performance is better, and robots based on it can also plan well in various environments.



Fig. 9. SMINPA-based dynamic planning in the uncertain environment 1.

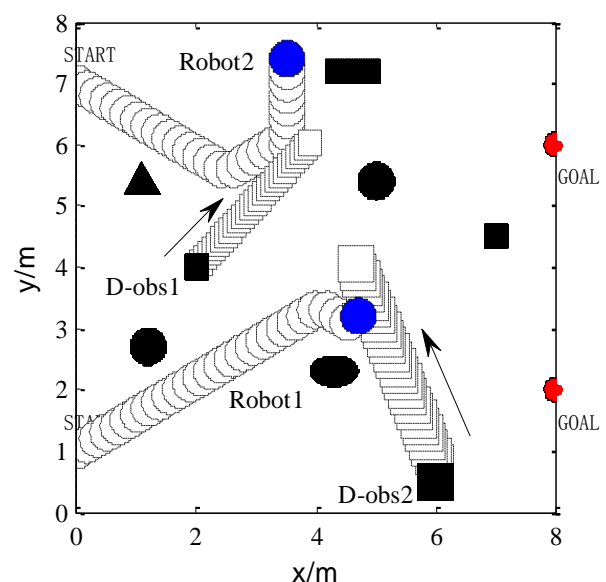

Fig. 10. SMINPA-based dynamic planning in the uncertain environment 2.

\section{Conclusion}

In order to better solve the path planning problem of multi-service robots in various environments, an improved measurement algorithm based on the mutual-coupled immune network planning algorithm is proposed. Antibodies are redefined according to the detection distance and the weight coefficient is defined dynamically according to the distance of the robot apart from the obstacles and the target, which makes the DW-AMINPA own stronger global planning ability. From the test results of static environments, it can be seen that the length of planned path of the proposed algorithm is shorter, the average angle is smaller, and smoothness is better. From the test results of dynamic environments, robots can avoid dynamic obstacles very well and reach the target based on the proposed algorithm, thus the effectiveness of service robots adaptive mutual-coupled immune network planning algorithm based on the distance-weighted is confirmed.

\section{References}

[1] Zhang, L. J. (2012). Path Planning Method Research for Autonomous Mobile Service Robot. Tianjin University of Science and Technology.

[2] Zhang, J. G., He, Y. Y., Fang, M. L., et al. (2011). Multi-service robot path planning based on improved ant colony algorithm. Mechanical and Electrical Engineering, 28(4), 448-452.

[3] Ying, Y. Y., Jin, C., \& Wang, S. G. (2013). Intelligent robot path planning algorithm research based on the 
indoor environment. Guangdong Communication Technology, (11), 36-41.

[4] Shi, J., Qing, \& H. W. (2015). Multi-robot path planning based on the optimal detection. Industrial Control Computer, (2), 71-72.

[5] Wang, L. J. (2012). Practical Path Planning Method Research on Multi-tasking Robot in the Supermarket. Shenyang University of Technology.

[6] Zheng, T. X., \& Wang, P. (2004). Multi-robot coordinated path planning in unknown dynamic environment. Proceedings of High-Level Forum on Global Manufacture \& Conference on Simulation Technology in the 21st Century (pp. 388-391).

[7] Sánchez-Ante, G., Ramos, F., \& Frausto, J. (2000). Cooperative Simulated Annealing for Path Planning in Multi-robot Systems. Proceedings of MICAI 2000: Advances in Artificial Intelligence. Springer Berlin Heidelberg (pp. 148-157).

[8] Hao, W., \& Qin, S. (2011). Multi-objective Path Planning for Space Exploration Robot Based on Chaos Immune Particle Swarm Optimization Algorithm. Artificial Intelligence and Computational Intelligence. Springer Berlin Heidelberg, 42-52.

[9] Das, P. K., Pradhan, S. K., Patro, S. N., et al. (2012). Artificial Immune System Based Path Planning of Mobile Robot. Soft Computing Techniques in Vision Science. Springer Berlin Heidelberg, 195-207.

[10] Pan, J., Wang, X. S., \& Cheng, Y. H. (2012). Mobile robot path planning based on improved ant colony algorithm. Journal of China University of Mining and Technology, 41(01), 108-113.

[11] Wang, S. A., Zhuang, J. (2002). Mobile robot path finding and planning algorithm based on the artificial immune network. Journal of System Simulation, 14(8), 995-997.

[12] Ishiguro, A., Watanabe, Y., \& Uchikawa, Y. (1995). An immunological approach to dynamic behavior control for autonomous mobile robots. Proceedings of IEEE/RSJ International Conference on Intelligent Robots and Systems 95. 'Human Robot Interaction and Cooperative Robots', Proceedings. (pp.495-500).

[13] Li, Y. (2007). Research and Design of Navigation System of Outdoor Autonomous Mobile Robot. QingDao, Ocean University of China.

[14] Yang, S. Q., \& Fu, W. P. (2008). Evolution research of intelligent robot navigation behavior based on the dynamic behavior. Journal of Applied Mechanics, 25(1), 66-70.

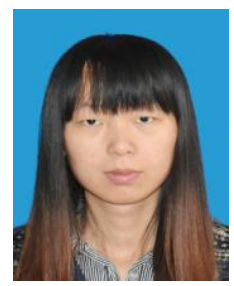

Rong Chen was born in Jiangsu, China, in 1993. She received her B.S. degree in Jiangsu University of Science and Technology at 2015. Currently, She is postgraduate student in the School of Mechanical Engineering at Nanjing University of Science and Technology, China. Her research interests mainly focus on electricity learning and data mining.

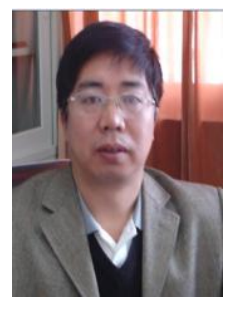

Hutian Feng was born in 1965. He received the D.E. degree in the School of Mechanical Engineering at Nanjing University of Science and Technology, China. Currently, he is a professor and the tutor of Dr. and master at Nanjing University of Science and Technology. His main research direction is Machinery and Electronics Engineering, and auxiliary research direction is precision instruments \& machinery. He has a number of academic posts and research projects. His research interests include information management, mechanical automation and data-mining. 